\title{
Hypoxia-Induced Suppression of Antiapoptotic Bcl-2 Expression in Human Bladder Tumor Cells Is Regulated by Caveolin-1-Dependent Adenosine Monophosphate-Activated Protein Kinase Activity
}

\author{
Tae Jin Cho ${ }^{1,2}$, Da-Hyun Lee ${ }^{1}$, Bo-Hwa Choi ${ }^{1}$, Helen K. Shinn ${ }^{3}$, Chang-Shin Park ${ }^{1}$ \\ ${ }^{1}$ Department of Pharmacology, Hypoxia-Related Disease Research Center, Inha Research Institute for Medical Sciences, Incheon, Korea \\ ${ }^{2}$ Department of Emergency Medicine, College of Medicine, Catholic Kwandong University, International St. Mary's Hospital, Incheon, Korea \\ ${ }^{3}$ Department of Anesthesiology and Pain Medicine, Inha University College of Medicine, Incheon, Korea
}

Purpose: Adenosine monophosphate-activated protein kinase (AMPK) is thought to inhibit cell proliferation or promote cell death, but the details remain unclear. In this study, we propose that AMPK inhibits the expression of anti-apoptotic B-cell lymphoma 2 (Bcl-2) by relying on the hypoxia-inducible factor 1 alpha (HIF-1 $\alpha$ )-induced caveolin-1 (Cav-1) expression pathway in noninvasive human bladder tumor (RT4) cells.

Methods: In cells exposed to a hypoxic environment (0.5\% oxygen), the levels of expression and phospho-activity of the relevant signaling enzymes were examined via Western blots and reverse transcription-polymerase chain reaction. Cell proliferation was assessed using a Cell Counting Kit-8 assay.

Results: The level of expression of Cav-1 was very low or undetectable in RT4 cells. Hypoxia was associated with significantly decreased cell growth, along with marked induction of HIF-1 $\alpha$ and Cav-1 expression; additionally, it suppressed the expression of the antiapoptotic marker Bcl-2 while leaving AMPK activity unchanged. Under hypoxic conditions, HIF-1 $\alpha$ acts as a transcription factor for Cav-1 mRNA gene expression. The cell growth and Bcl-2 expression suppressed under hypoxia were reversed along with decreases in the induced HIF- $1 \alpha$ and Cav-1 levels by AMPK activation with metformin $(1 \mathrm{mM})$ or phenformin $(0.1 \mathrm{mM})$. In addition, pretreatment with AMPK small interfering RNA not only increased the hypoxia-induced expression of HIF-1 $1 \alpha$ and Cav-1, but also reversed the suppression of Bcl-2 expression. These results suggest that HIF-1a and Cav-1 expression in hypoxic environments is regulated by basal AMPK activity; therefore, the inhibition of Bcl-2 expression cannot be expected when AMPK activity is suppressed, even if Cav-1 expression is elevated.

Conclusions: For the first time, we find that AMPK activation can regulate HIF-1 $\alpha$ induction as well as HIF-1 $\alpha$-induced Cav1 expression, and the hypoxia-induced inhibitory effect on the antiapoptotic pathway in RT4 cells is due to Cav-1-dependent AMPK activity.

Keywords: Hypoxia; AMP-activated protein kinases; Caveolin-1; Bcl-2; Human bladder tumor cells; Noninvasive RT4 cells

- Fund/Grant Support: This work was supported by grants from the National Research Foundation of Korea (NRF) funded by the Korean government (MSIP) (NRF-2014R1A5A2009392 to C.-S. Park), as well as the Basic Science Research Program through the National Research Foundation of Korea (NRF), funded by the Ministry of Education (NRF-2020R1I1A1A01065009 to B.-H. Choi).

- Conflict of Interest: No potential conflict of interest relevant to this article was reported.

Corresponding author: Chang-Shin Park (i) https://orcid.org/0000-0001-6519-2857 Department of Pharmacology, Hypoxia-Related Disease Research Center, Inha Research Institute for Medical Sciences, Inha University College of Medicine, 100 Inha-ro, Michuhol-gu, Incheon 22212, Korea

Email: parkshin@inha.ac.kr

Co-corresponding author: Bo-Hwa Choi (iD https://orcid.org/0000-0002-7907-8059

Department of Pharmacology, Hypoxia-Related Disease Research Center, Inha

Research Institute for Medical Sciences, Inha University College of Medicine,

100 Inha-ro, Michuhol-gu, Incheon 22212, Korea

Email: bohwa813@gmail.com

Submitted: November 18, 2020 / Accepted after revision: January 28, 2021
This is an Open Access article distributed under the terms of the CrecC.
commons.org/licenses/by-nc/4.0/) which permits unrestricted non-commercial use, distribution, and reproduction in any medium, provided the original work is properly cited. 


\begin{abstract}
- HIGHLIGHTS
- AMPK inhibits the expression of antiapoptotic Bcl-2 by relying on the HIF-1a-induced Cav-1 expression pathway in Cav-1-free RT4 bladder tumor cells, and the hypoxia-induced inhibitory effect on the anti-apoptotic pathway is due to Cav-1-dependent AMPK activity. These findings point to the interactions between AMPK and Cav-1 impacting Bcl-2 expression.
\end{abstract}

\section{INTRODUCTION}

Hypoxia is known to affect various signals related to tumor aggression, prognosis, and metastasis [1-4]. Hypoxia can be one of the most potent inducers of gene expression for pathways related to oxygen $\left(\mathrm{O}_{2}\right)$ sensation [5]. Hypoxia-induced factor 1 (HIF-1) is a major transcriptional regulatory factor in hypoxiainduced processes. HIF-1 is a heterodimer consisting of HIF-1a and HIF-1a subunits, and insufficient levels of $\mathrm{O}_{2}$ result in the overexpression of HIF-1 $\alpha$ [6-8].

Adenosine monophosphate-activated protein kinase (AMPK) is a serine/threonine protein kinase that regulates cellular energy status and differentiation and responds to metabolic stress $[9,10]$. Additionally, one of its major functions is the regulation of cell proliferation. AMPK is a heterotrimeric enzyme consisting of a catalytic $\alpha$ subunit and regulatory $\beta$ and $\gamma$ subunits, present in several isoforms. It is activated by various cellular stresses that increase cell AMP/adenosine triphosphate ratios, such as glucose deficiency, exercise, and hypoxia [11]. AMPK is known to be activated in hypoxic environments $[12,13]$, but the changes in HIF-1 $\alpha$ and HIF-2 expression after AMPK activation are still unclear $[14,15]$.

In addition to hypoxia-induced AMPK activation, hypoxia induces the differential expression of caveolin-1 (Cav-1), a cellular membrane scaffolding protein, in several cell types [1618]. Cav-1 is a major structural component of caveolae, which are flask-shaped vesicular invaginations of the plasma membrane. Cav-1 plays an important functional role in the transport of cellular lipids, the regulation of cell proliferation, cell death involving apoptosis, adhesion, and the invasion of tumor cells [19]. In particular, the role of Cav-1 in human tumor progression remains a subject of debate, as it is uncertain whether Cav1 promotes cell survival and proliferation or induces apoptosis as a tumor suppressor [20]. In order to better control tumor cell proliferation and progression, the role of Cav-1 in apoptosis should be clarified. In human bladder cancer, Cav-1 expression is correlated with tumor stage and grade. Low or absent Cav-1 expression has been observed in noninvasive (or superficial) human bladder epithelial cells (RT4 cells), whereas its expression is high in aggressive (or invasive) human bladder epithelial cells (T24 cells) [1,3,4,21,22].

In this study, we demonstrated that Cav-1 expression was induced via a transcriptional pathway of HIF-1a in hypoxia-exposed RT4 cells, and hypoxia-induced inhibition of cell growth as indicated by a marked decrease in B-cell lymphoma 2 (Bcl-2) expression was induced by basal AMPK activity only in the presence of Cav-1 expression. Furthermore, we report that AMPK activation eliminated or reversed the hypoxia-related induction of HIF- $1 \alpha$ and associated Cav- 1 expression and also reversed the effect on Bcl-2 expression. These findings point to possible interactions between AMPK and Cav-1 impacting Bcl2 expression.

\section{MATERIALS AND METHODS}

\section{Materials}

Phenformin hydrochloride, 1,1-dimethylbiguanide hydrochloride (metformin), echinomycin, and $\beta$-actin antibody were purchased from Sigma-Aldrich (St. Louis, MO, USA). Antibodies for AMPKa, phospho-AMPKa (Thr172), phosphoAMPKal (Ser485), acetyl-CoA carboxylase, and phospho-acetyl-CoA carboxylase (Ser79) were purchased from Cell Signaling Technology (Beverly, MA, USA), and antibodies for Cav-1 and Bcl-2 as well as AMPKa1/2 small interfering RNA (siRNA) and control siRNA were purchased from Santa Cruz Biotechnology (Santa Cruz, CA, USA). HIF-1 $\alpha$ antibody was obtained from R\&D Systems (Minneapolis, MN, USA).

\section{Cell Culture}

RT4 cells (Korean Cell Line Bank, Seoul, Korea) were cultured in Dulbecco's modified Eagle medium (Gibco, El Paso, TX, USA) containing $10 \%$ fetal bovine serum (genDEPOT, Barker, TX, USA) and 1\% penicillin-streptomycin (Gibco), while T24 cells (Korean Cell Line Bank, Seoul, Korea) were cultured in RPMI 1640 medium (Gibco) containing 10\% fetal bovine serum (genDEPOT), 1\% penicillin-streptomycin (Gibco), and 20mM HEPES (Sigma-Aldrich). These cells were serially passaged in the laboratory using $0.25 \%$ trypsin-EDTA (Gibco). The cells were grown at $37^{\circ} \mathrm{C}$ in a humidified atmosphere of $5 \%$ carbon dioxide $\left(\mathrm{CO}_{2}\right)$ and $95 \%$ air. For all experiments, cells 
were seeded into $60-\mathrm{mm}$ plates overnight and then treated with relevant reagents or vehicle only for the indicated time.

\section{Hypoxic Conditions}

Hypoxic conditions $\left(0.5 \% \mathrm{O}_{2}\right.$ and $5 \% \mathrm{CO}_{2}$ balanced with $\mathrm{N}_{2}$ ) were achieved in a humidified aerobic workstation (InvivO $\mathrm{O}_{2}$ 500 with $\mathrm{I}-\mathrm{CO}_{2} \mathrm{~N}_{2} \mathrm{IC}$ gas mixing system; The Baker Company, Sanford, ME, USA), which contained an oxygen sensor that continuously monitored the chamber oxygen tension.

\section{Western Blot Analysis}

For whole-cell extracts, cells were washed with ice-cold phosphate-buffered saline and collected by scraping in ice-cold NOS lysis buffer (320mM sucrose, 200mM HEPES, and 1mM EDTA; $\mathrm{pH}, 7.2$ ) supplemented with Phos Stop (Roche, Mannheim, Germany) and protease inhibitor (Sigma-Aldrich). The harvested cells were sonicated 5 times for 5 seconds each at $3 \mathrm{~W}$ and centrifuged at 3,000 rpm, 10 minutes, and $4^{\circ} \mathrm{C}$. The supernatant was quantified using the Pierce BCA Protein Assay Kit (Pierce, Rockford, IL, USA). An equal amount of protein for each blot was loaded on a 4\%-20\% Mini-PROTEAN TGX Precast Protein Gel (BIO-RAD, Hercules, CA, USA), transferred to a polyvinylidene difluoride membrane (Millipore, Bedford, MA, USA), and then blocked with 5\% nonfat milk in tris-buffered saline with $0.05 \%$ tween 20 (TBS-T) buffer. The membrane was incubated with the indicated antibody overnight at $4^{\circ} \mathrm{C}$ and was then washed 3 times in TBS-T buffer. After incubation with the secondary antibody, detection was performed with $\mathrm{Su}$ perSignal West Pico Chemiluminescent Substrate (Thermo Scientific, Rockford, IL, USA).

\section{Determination of Cell Proliferation via Cell Counting Kit-8 (CCK-8) Assay}

Cell viability and proliferation were measured with a Cell Counting Assay Kit-8 (Dojindo, Rockville, MD, USA). Trypsinized cells were resuspended in Dulbecco's modified Eagle medium at $1 \times 10^{5}$ cells $/ \mathrm{mL}$, and $100 \mu \mathrm{L}$ of cell suspension was distributed into each well of a 96-well flat-bottom microplate. Following incubation to allow adherent cell growth, the medium was removed, and $100 \mu \mathrm{L}$ of fresh medium was distributed into each well and incubated in a $\mathrm{CO}_{2}$ incubator $\left(37^{\circ} \mathrm{C}\right.$, with a humidified atmosphere of $5 \% \mathrm{CO}_{2}$ and $95 \%$ air) or a hypoxia chamber $\left(0.5 \% \mathrm{O}_{2}\right.$ and $5 \% \mathrm{CO}_{2}$ balanced with $\left.\mathrm{N}_{2}\right)$ for $6,12,24$, and 48 hours. After incubation, $10 \mu \mathrm{L}$ of CCK- 8 solution was added to each well of the plate and incubated for 2 hours in the incubator. The absorbance on the plate was then measured at $450 \mathrm{~nm}$ with a microplate reader.

\section{Treatment With AMPK siRNA}

Cells were washed with Opti-MEM medium (Gibco) and transfected with 10 pmol control or AMPK siRNA using Lipofectamine 2000 (Invitrogen, Carlsbad, CA, USA) according to the manufacturer's protocol. The medium was replaced with growth medium 24 hours after transfection, and the cells were incubated overnight for stabilization. After changing to fresh medium, the cells were incubated in a $\mathrm{CO}_{2}$ incubator $\left(37^{\circ} \mathrm{C}\right.$, with a humidified atmosphere of $5 \% \mathrm{CO}_{2}$ and $95 \%$ air) or a hypoxia chamber $\left(0.5 \% \mathrm{O}_{2}\right.$ and $5 \% \mathrm{CO}_{2}$ balanced with $\left.\mathrm{N} 2\right)$ for 24 and 48 hours. Protein expression was evaluated using Western blotting.

\section{RNA Extraction and cDNA Synthesis}

The total RNA was isolated using AccuZol Total RNA Extraction Reagent (Bioneer Corporation, Daejeon, South Korea) according to the manufacturer's instructions. cDNA was synthesized using the AccuPower CycleScript RT Premix ( $\mathrm{dT}_{20}$; Bioneer), and the synthesis was carried out as follows: 40 cycles for 1 minute at $15^{\circ} \mathrm{C}, 4$ minutes at $50^{\circ} \mathrm{C}, 1$ cycle for 5 minutes at $95^{\circ} \mathrm{C}, 60$ minutes at $50^{\circ} \mathrm{C}$, and 5 minutes at $95^{\circ} \mathrm{C}$.

\section{Reverse Transcription-Polymerase Chain Reaction Analysis} Reverse transcription-polymerase chain reaction (RT-PCR) was performed with Solg e-Taq DNA Polymerase (10X e-Taq Reaction Buffer, 10mM dNTP mix, Solg e-Taq), sense and antisense primers, and a cDNA template with the following protocol: 1 cycle for 2 minutes at $95^{\circ} \mathrm{C}, 35$ cycles for 20 seconds at $95^{\circ} \mathrm{C}, 40$ seconds at $55^{\circ} \mathrm{C}, 1$ minute at $72^{\circ} \mathrm{C}$, and 1 cycle for 5 minutes at $72^{\circ} \mathrm{C}$. RT-PCR primers were used for Cav-1 (sense, 5'-ACG TAG ACT CGG AGG GAC ATC TCT-3'; antisense, 5'-CTG CAA GTT GAT GCG GAC ATT GC- ${ }^{\prime}$ ) and glyceraldehyde 3-phosphate dehydrogenase (sense, 5'-TCA TTG ACC TCA ACT ACA TGG T-3'; antisense, 5'-CTA AGC AGT TGG TGG TGC AG-3'). The samples were then analyzed via $2 \%$ agarose gel electrophoresis with $0.5 \%$ tris-borate-EDTA buffer.

\section{Statistical Analyses}

The statistical analysis was performed with GraphPad Prism software ver. 9 (GraphPad Software, San Diego, CA, USA). Data were represented as the mean \pm standard error of the mean of 3 or more independent experiments. Based on the Western 
blots, differences between groups (normoxia and hypoxia) and within groups (siRNA-treated and control for each condition) were assessed using the unpaired Student $t$-test. For the between-group comparisons, $\mathrm{P}$-values of ${ }^{\star} \mathrm{P}<0.05,{ }^{*} \mathrm{P}<0.01$, and ${ }^{* * *} \mathrm{P}<0.001$ were considered to indicate statistical significance, while $\mathrm{P}$-values of ${ }^{*} \mathrm{P}<0.05$ and ${ }^{* *} \mathrm{P}<0.01$ were considered to do so for the within-group comparisons. Two-way analysis of variance, followed by the Tukey multiple comparison test, was used as the statistical test for cell proliferation levels ( ${ }^{* *} \mathrm{P}<0.001$ between the normoxia and hypoxia groups).

\section{RESULTS}

\section{Hypoxia-Induced AMPK Activation and Cav-1 Expression}

AMPK is involved in the regulation of cell survival or proliferation. The purpose of this study was to examine the changes in the expression and activity of AMPK in urothelial cells after exposure to a hypoxic environment $\left(0.5 \% \mathrm{O}_{2}\right)$. As an initial step, the level of expression of Cav-1 and the activity of Src kinase (a tyrosine kinase) was compared between noninvasive urothelial (RT4) cells and high-grade invasive urothelial carcinoma (T24) cells. In RT4 cells, the expression of Cav-1 was almost too low to be detected. In contrast, the activity of Src kinase (Tyr416) was much higher in RT4 cells than in T24 cells (data not shown).

In the present study, the levels of expression of HIF-1 $\alpha$ ( 48 hours, ${ }^{*} \mathrm{P}=0.002$ ) and Cav-1 (24 hours, ${ }^{*} \mathrm{P}=0.049 ; 48$ hours, ${ }^{\star} \mathrm{P}=0.038$ ) were markedly greater in RT4 cells exposed to hypoxia than in the cells treated with normal oxygen concentrations (Fig. 1). Of particular note, the level of AMPK activity was unchanged (as confirmed by the p-ACC/ACC ratio), while a decrease in AMPK expression was shown by reduced activity at the p-AMPK-172 activation site and the p-AMPK-485 inhibition site ( 24 hours, ${ }^{*} \mathrm{P}=0.017$ and 48 hours, ${ }^{*} \mathrm{P}=0.044$ vs. normoxia). Although not shown in these results, AMPK phosphorylation activity was weakly elevated in a time-dependent manner at the early time points (1, 4, and 24 hours) of hypoxia exposure. In addition, the expression of Bcl-2, an anti-apoptotic signaling enzyme, was significantly lower under hypoxic conditions than normal oxygen conditions ( 24 hours, ${ }^{*} \mathrm{P}=0.014$ and 48 hours, ${ }^{\star} \mathrm{P}=0.036$ ) (Fig. 1). These results suggest that RT4 cells may exhibit inhibited cell proliferation or induced apoptosis under hypoxic conditions. However, the changes observed in the RT4 cell line under hypoxic conditions were close to nonexistent in the T24 cell line, although no statistical analysis was conducted (Fig. 1).

\section{Determination of the Cell Proliferation Rate In Hypoxic Conditions}

To elucidate the impact of hypoxia on RT4 cell proliferation, the cells in the normoxic and hypoxic conditions were quantified over time with the CCK-8 assay. The doubling time of the cells was approximately 36 hours in normoxic conditions. At the early time point ( 12 hours), no obvious difference was observed between normoxic and hypoxic conditions. In hypoxic conditions, the cell proliferation rate was significantly lower than in normoxic conditions at both 24 and 48 hours ( 35\% lower at 24 hours, ${ }^{* * *} \mathrm{P}<0.001 ; \sim 70 \%$ lower at 48 hours, ${ }^{* * *} \mathrm{P}<0.001$; Fig. 2 ). These results suggest that in RT4 cells, cell proliferation may be significantly reduced or cell death may be induced under hypoxia relative to normoxia $\left({ }^{* * *} \mathrm{P}<0.001\right)$.

\section{Effects of the AMPK Activators Metformin and Phenformin on Hypoxia-Induced HIF-1a, Cav-1, and Bcl-2 Expression}

Under hypoxic conditions, the expression of HIF-1a was significantly induced relative to normoxic conditions at both 24 and 48 hours ( ${ }^{* *} \mathrm{P}=0.005$ and ${ }^{* *} \mathrm{P}=0.007$, respectively), while the expression of Cav-1 was significantly induced only at 48 hours $\left({ }^{\star} \mathrm{P}=0.027\right)$. The levels of AMPK activity, as well as the p-ACC/ ACC ratio, were unchanged or slightly increased, with a decrease in AMPK expression, a decrease in p-AMPK-172 phospho-activity, and a significant decrease in $\mathrm{p}$-AMPK-485 phospho-activity ( $\left.{ }^{\star} \mathrm{P}=0.019\right)$ relative to normoxia. In contrast, the expression of Bcl-2 was reduced to approximately $60 \%$ of its level under normoxia ( 24 hours, ${ }^{*} \mathrm{P}=0.004 ; 48$ hours, ${ }^{* *} \mathrm{P}=$ 0.002 ) (Fig. 3). The differences in the expression and activity levels of these enzymes under hypoxia were nonsignificantly (within the hypoxia group) reversed, along with a significant decrease in the expression of HIF-1 $\alpha$, by pretreatment with the AMPK activators metformin $\left({ }^{*} \mathrm{P}=0.011\right.$ and ${ }^{* *} \mathrm{P}=0.001$ vs. normoxia $)$ and phenformin $\left({ }^{\star} \mathrm{P}=0.021\right.$ and ${ }^{\star} \mathrm{P}=0.029$ vs. normoxia) (Fig. 3). These results may imply that the inducible expression of HIF-1a, a transcriptional regulator in hypoxic conditions, has the same tendency as the induction of Cav-1 expression, while that of Bcl-2 expression, which suppresses apoptosis, opposes the results for HIF-1 $\alpha$ and Cav-1. In addition, overactivity of AMPK may reduce the induction of HIF$1 \alpha$ expression or stability and suppress HIF-1 $1 \alpha$-induced Cav-1 expression, thus regulating a series of HIF-1a-/Cav-1-mediated apoptosis signaling pathways. 

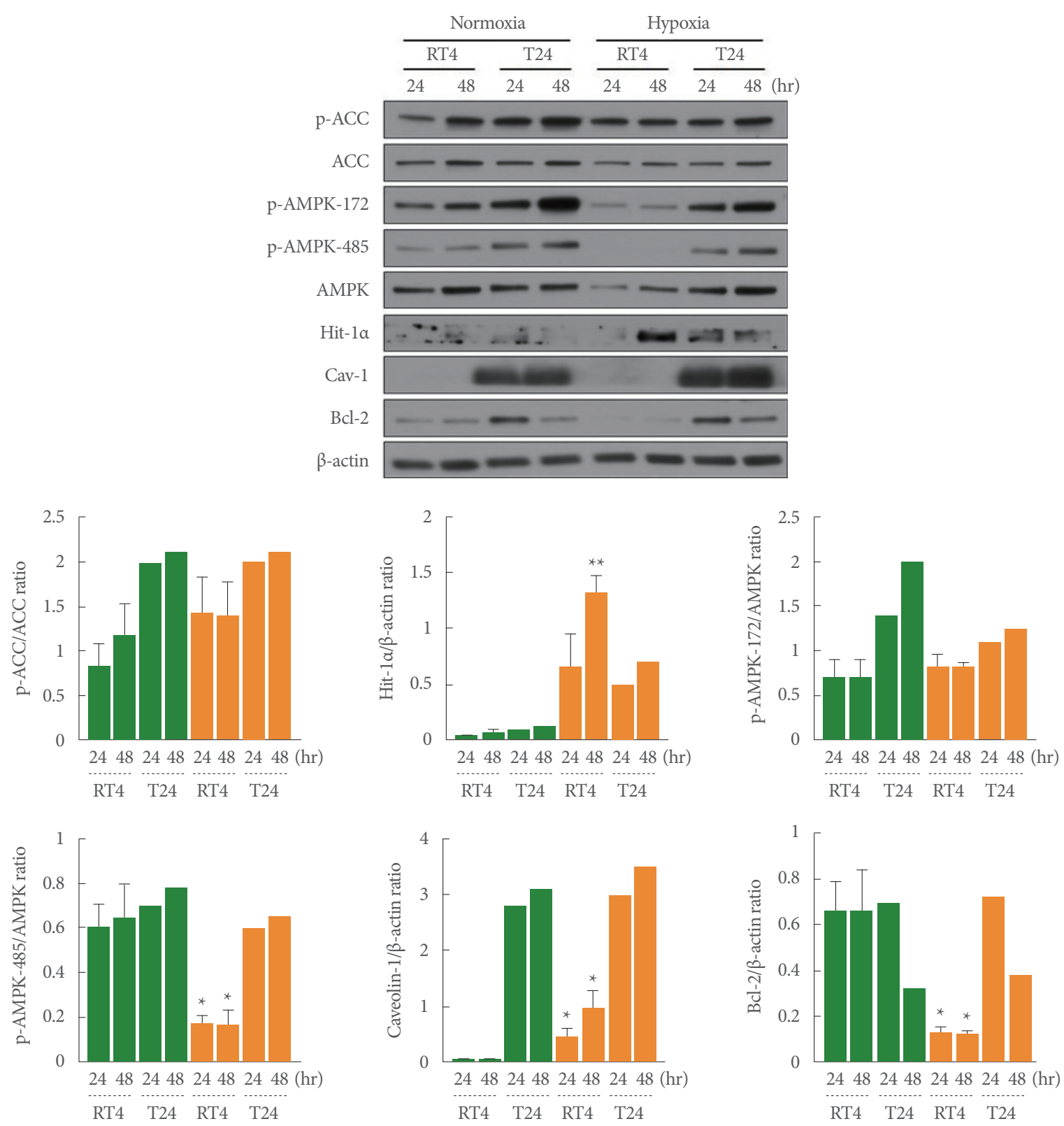

Fig. 1. Basal and induced expression and/or phospho-activity of acetyl-CoA carboxylase (ACC), adenosine monophosphate-activated protein kinase (AMPK), hypoxia-inducible factor-1 (HIF-1a), caveolin-1 (Cav-1), and B-cell lymphoma 2 (Bcl-2) under normoxia and hypoxia in noninvasive superficial (RT4) and high-grade invasive (T24) human bladder tumor cells at 24 hours and 48 hours. pAMPK-172 and p-AMPK-485 refer to the stimulatory and inhibitory phosphorylation sites, respectively. In particular, substantial AMPK activity can be predicted based on the ratio of p-ACC/ACC. Black and grey bars indicate the normoxia and hypoxia groups, respectively. Values are expressed as the mean \pm standard error of the mean. ${ }^{*} \mathrm{P}<0.05$ and ${ }^{* *} \mathrm{P}<0.01$ vs. normoxia (RT4 cells; statistical analysis was not performed for T24 cells).

\section{Effects of the AMPK Activators Metformin and Phenformin on Hypoxia-Induced Cav-1 mRNA Expression}

The levels of Cav-1 mRNA expression were assessed via RTPCR at 6, 12, and 24 hours to determine whether hypoxia-induced Cav-1 expression was induced at the transcriptional lev- el. As shown in Fig. 4, Cav-1 mRNA expression increased in a time-dependent manner under hypoxic conditions, and this increase in Cav-1 mRNA was inhibited in both of the AMPK activator pretreatment conditions ( $1 \mathrm{mM}$ metformin and $0.1 \mathrm{mM}$ phenformin). This suggests that the hypoxia-induced 


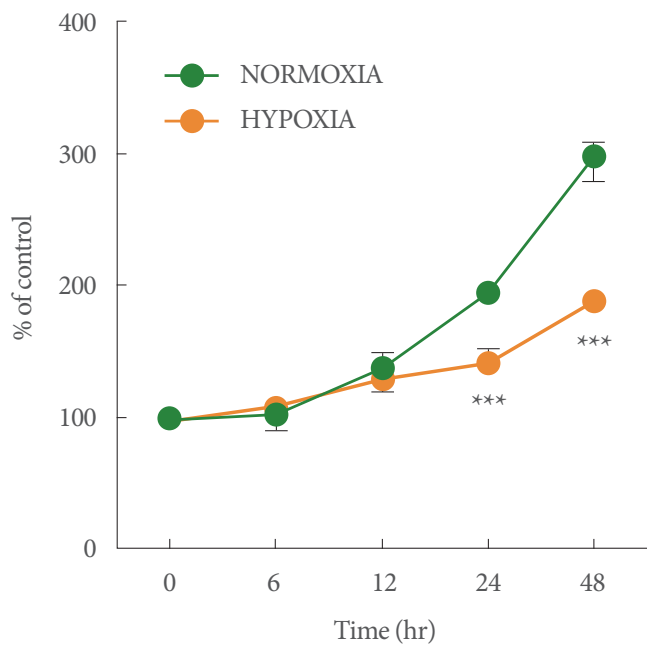

Fig. 2. Cell proliferation over time under normoxic and hypoxic conditions. The cells were counted at baseline and at 4 time points $(0,6,12,24$, and 48 hours). Each value represents the mean \pm standard error of the mean of 5 independent experiments. The difference between the normoxia and hypoxia groups was analyzed via 2-way analysis of variance with the Tukey multiple comparison test. ${ }^{* *} \mathrm{P}<0.001$ vs. normoxia ( 24 hours and 48 hours).

expression of Cav-1 occurs at the mRNA level and that excessive AMPK activity can regulate the Cav-1 transcriptional pathway, although a statistical analysis was not performed.

\section{Effects of the HIF-1a-Specific Inhibitor Echinomycin on the Induction of Cav-1 mRNA in Cells Exposed to Normoxia and Hypoxia}

To demonstrate that HIF-1 $\alpha$ acts as a transcription factor for Cav-1 induction, cell lines exposed to hypoxia were pretreated with echinomycin, a selective inhibitor of HIF-1 $\alpha$. As shown in Fig. 5, Cav-1 mRNA expression increased in a time-dependent and gradual fashion under hypoxic conditions, but Cav-1 mRNA induction was suppressed in a similarly time-dependent manner by echinomycin $(2 \mathrm{nM})$ pretreatment. This result suggests that hypoxia-induced HIF- $1 \alpha$ acts as a transcription factor of Cav-1 mRNA gene expression, although no statistical analysis was conducted.

\section{Effects of Echinomycin on Hypoxia-Induced Cav-1 Expression}

To confirm whether the HIF-1 $\alpha$ transcription factor is involved in hypoxia-induced Cav-1 expression, we pretreated the cells under the hypoxic conditions with echinomycin (1nM and $2 \mathrm{nM}$ ), a selective inhibitor of HIF-1 $\alpha$. The cytotoxicity associat- ed with echinomycin was measured via the CCK-8 assay, and HIF-1a expression completely disappeared in the echinomycinpretreated hypoxic condition relative to the untreated hypoxic cells ( ${ }^{*} \mathrm{P}=0.025$ and ${ }^{*} \mathrm{P}=0.023$ ) (Fig. 6). The increased expression of Cav-1 induced in hypoxic conditions under echinomycin-free conditions $\left({ }^{*} \mathrm{P}=0.004\right.$ vs. normoxia) was also reduced in echinomycin-pretreated conditions at 48 hours only $\left({ }^{*} \mathrm{P}=\right.$ 0.050) (Fig. 6). This indicates that the induction of Cav-1 mRNA and protein expression is mediated by the HIF-1 $\alpha$ transcription factor. However, the pretreatment of echinomycin ( $2 \mathrm{nM})$ under both normoxia $\left({ }^{*} \mathrm{P}=0.018\right.$ and ${ }^{*} \mathrm{P}=0.028$ vs. the untreated control at 24 and 48 hours, respectively) and hypoxia ${ }^{(} \mathrm{P}=0.011$ and ${ }^{\#} \mathrm{P}=0.027$ vs. the untreated control at 24 and 48 hours, respectively) showed a similar decrease in Bcl-2 expression, indicating echinomycin-induced activation of the apoptosis pathway of RT4 cell regardless of the oxygen condition.

\section{Effects of AMPK siRNA on Hypoxia-Induced HIF-1 $1 a$ and Cav-1 Expression}

A transient knockdown with AMPK siRNA was applied to demonstrate that AMPK activity plays a role in the regulatory pathway of hypoxia-induced HIF-1 $\alpha$ and Cav-1 expression. As shown in Fig. 7, AMPK-siRNA treatment significantly or nonsignificantly decreased AMPK expression under both normoxia $\left({ }^{*} \mathrm{P}=0.023\right.$ vs. the untreated control) and hypoxia $\left({ }^{*} \mathrm{P}=0.020\right.$ vs. the untreated control). As expected, the hypoxia-induced expression of HIF- $1 \alpha\left({ }^{*} \mathrm{P}=0.009\right.$ vs. normoxia) and Cav-1 (nonsignificant compared with normoxia) increased after AMPKsiRNA treatment $\left({ }^{* *} \mathrm{P}=0.003\right.$ and nonsignificant vs. the untreated control). This result suggests that AMPK activity can regulate the HIF-1 $\alpha /$ Cav-1 signaling pathway induced under hypoxic conditions. Surprisingly, the increased expression of Bcl-2 in AMPK siRNA-treated cells partially reversed the promotion of the hypoxia-induced apoptotic pathway in RT4 cells, as shown in Figs. 1, 3, and 6 (Fig. 7). Although this result cannot be completely explained with or without statistical significance, we posit that cellular apoptosis activity could not be exhibited because the AMPK activity was inhibited in the AMPK siRNA-treated cells.

\section{DISCUSSION}

In this study, we showed that HIF-1 $\alpha$-mediated Cav-1 expression was induced in Cav-1-free and noninvasive human bladder tumor (RT4) cells exposed to hypoxic conditions $\left(<0.5 \% \mathrm{O}_{2}\right)$. 

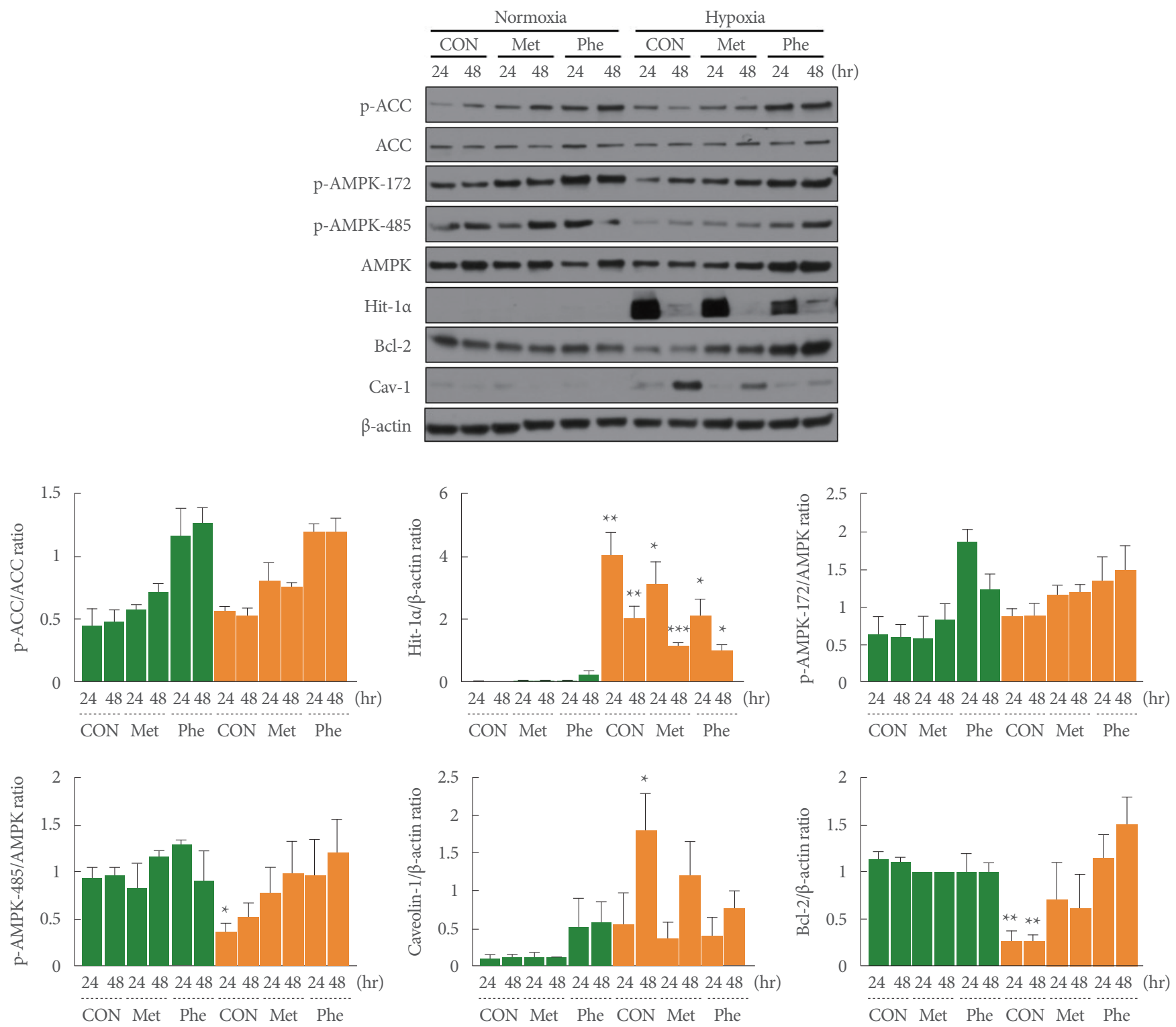

Fig. 3. Effects of the adenosine monophosphate-activated protein kinase (AMPK) activators metformin (Met) and phenformin (Phen) on the levels of expression and/or phospho-activity of acetyl-CoA carboxylase (ACC), AMPK, hypoxia-inducible factor-1 (HIF-1a), caveolin-1 (Cav-1), and B-cell lymphoma 2 (Bcl-2) under normoxia and hypoxia, at 24 hours and 48 hours. Hypoxia did not significantly affect either AMPK activity or the ratio of p-ACC/ACC, but it induced increases in both HIF- $1 \alpha\left({ }^{* *} \mathrm{P}<0.01\right)$ and Cav-1 $\left({ }^{*} \mathrm{P}<0.05\right)$ and, inversely, a decrease in $\mathrm{Bcl}-2\left({ }^{*} \mathrm{P}<0.01\right)$. These changes were reversed by pretreatment with Met or Phen, but nonsignificantly compared within the hypoxia group. The black and grey bars indicate the normoxia and hypoxia groups, respectively. The data presented are expressed as the mean \pm standard error of the mean.

Under these conditions, the activity of AMPK (as indicated by the p-ACC/ACC ratio), which has anti-cell proliferation or proapoptotic activity, was weakly increased or unchanged, while the expression of Bcl-2, an antiapoptotic signaling enzyme, was markedly decreased. That is, hypoxia appears to suppress the antiapoptotic Bcl-2 signaling pathway via an interactive mecha- nism between basal AMPK activity and Cav-1 expression.

The activation of AMPK under hypoxic conditions is well known [12,13,23], but a decrease [14,24,25] or increase [14] in HIF-1 $\alpha$ expression or stability caused by AMPK activation has been rarely reported and is still unclear. In this regard, a major finding of the present study is that excessive AMPK activation 

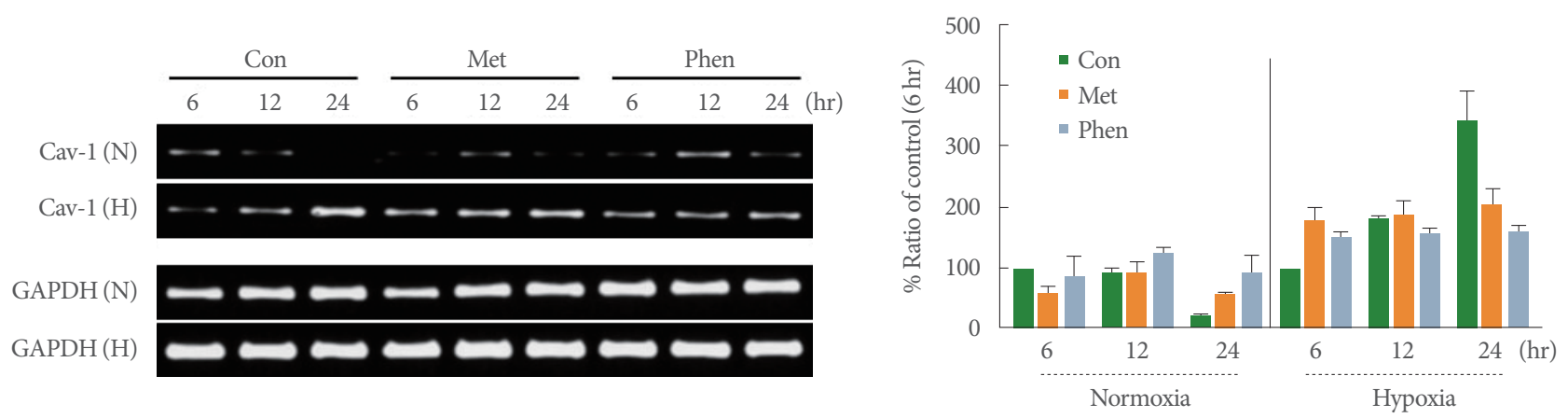

Fig. 4. Effects of the adenosine monophosphate-activated protein kinase (AMPK) activators metformin (Met) and phenformin (Phen) on caveolin-1 (Cav-1) mRNA induction under normoxic and hypoxic conditions at 6, 12, and 24 hours. Cav-1 mRNA expression was measured via real time-polymerase chain reaction. Time-dependent Cav-1 mRNA expression was induced by hypoxia (Con: control), but this was blocked by pretreatment with Met or Phen. The data presented are expressed as the mean \pm standard error of the mean of 2 independent experiments (\% ratio of control [6 hours]) without a statistical analysis.
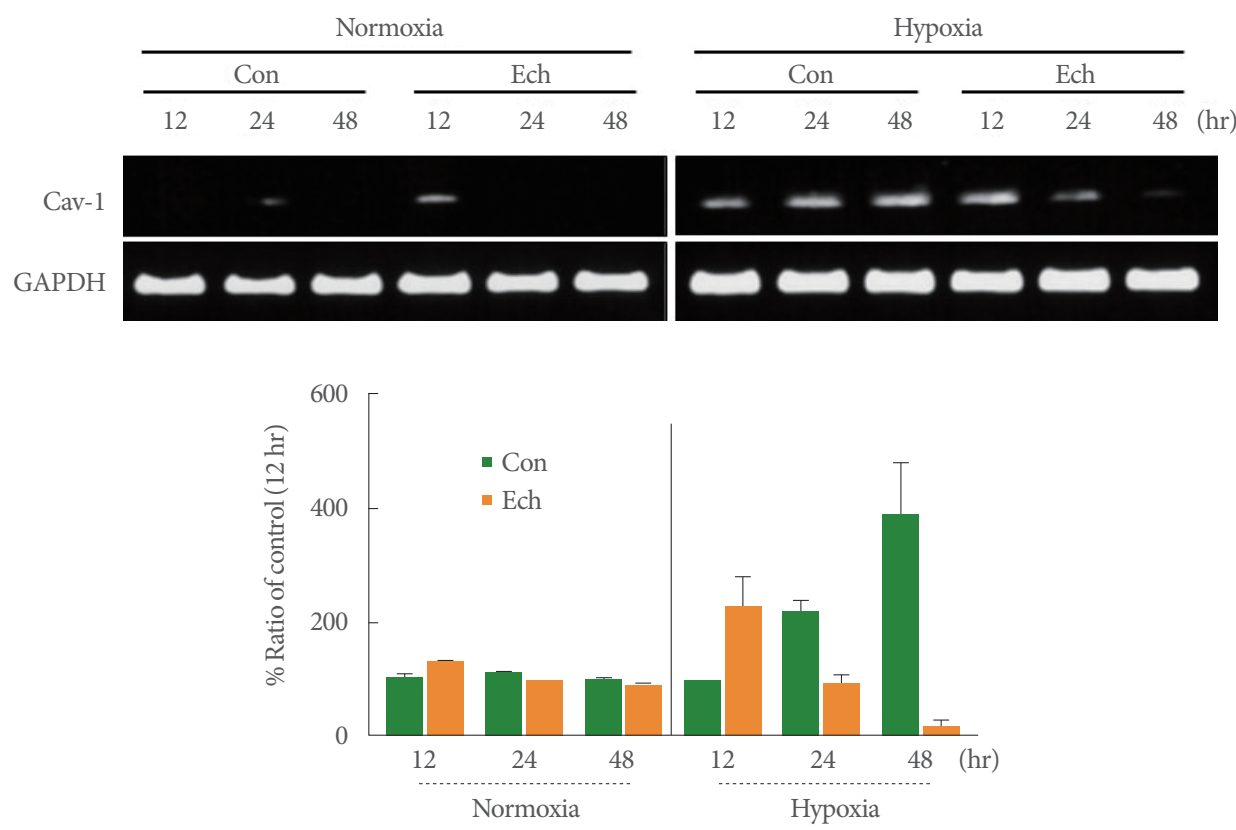

Fig. 5. Effects of the hypoxia-inducible factor 1 (HIF-1 $\alpha$ )-selective inhibitor echinomycin (Ech, 2nM) on caveolin-1 (Cav-1) mRNA induction under normoxia and hypoxia at 12,24, and 48 hours. Cav-1 mRNA expression was measured via reverse transcriptasepolymerase chain reaction. Ech pretreatment decreased the time-dependent hypoxia-induced Cav-1 mRNA expression. The data presented are expressed as the mean \pm standard error of the mean of 2 independent experiments (\% ratio of control [12 hours]) without a statistical analysis.

with metformin or phenformin under hypoxic conditions can regulate the induction of HIF-1 $\alpha$ itself or HIF- $1 \alpha$-induced Cav1 expression. This finding that the AMPK activity pathway can regulate Cav-1 expression is new, although it specifically occurs in RT4 cells under hypoxic conditions. Zhou et al. [26] demonstrated that the AMPK agonist metformin inhibited AMPK-independently hypoxia-induced HIF-1 $\alpha$ stability or accumula- tion.

More interestingly, in the present study, pretreatment with AMPK activators (metformin or phenformin) interfered with HIF- $1 \alpha$ and Cav- 1 induction, and this pretreatment suppressed the hypoxia-induced inhibition of cell growth or apoptosis while returning Bcl-2 expression to its normal level or increasing it. Therefore, in the RT4 cells exposed to hypoxic condi- 

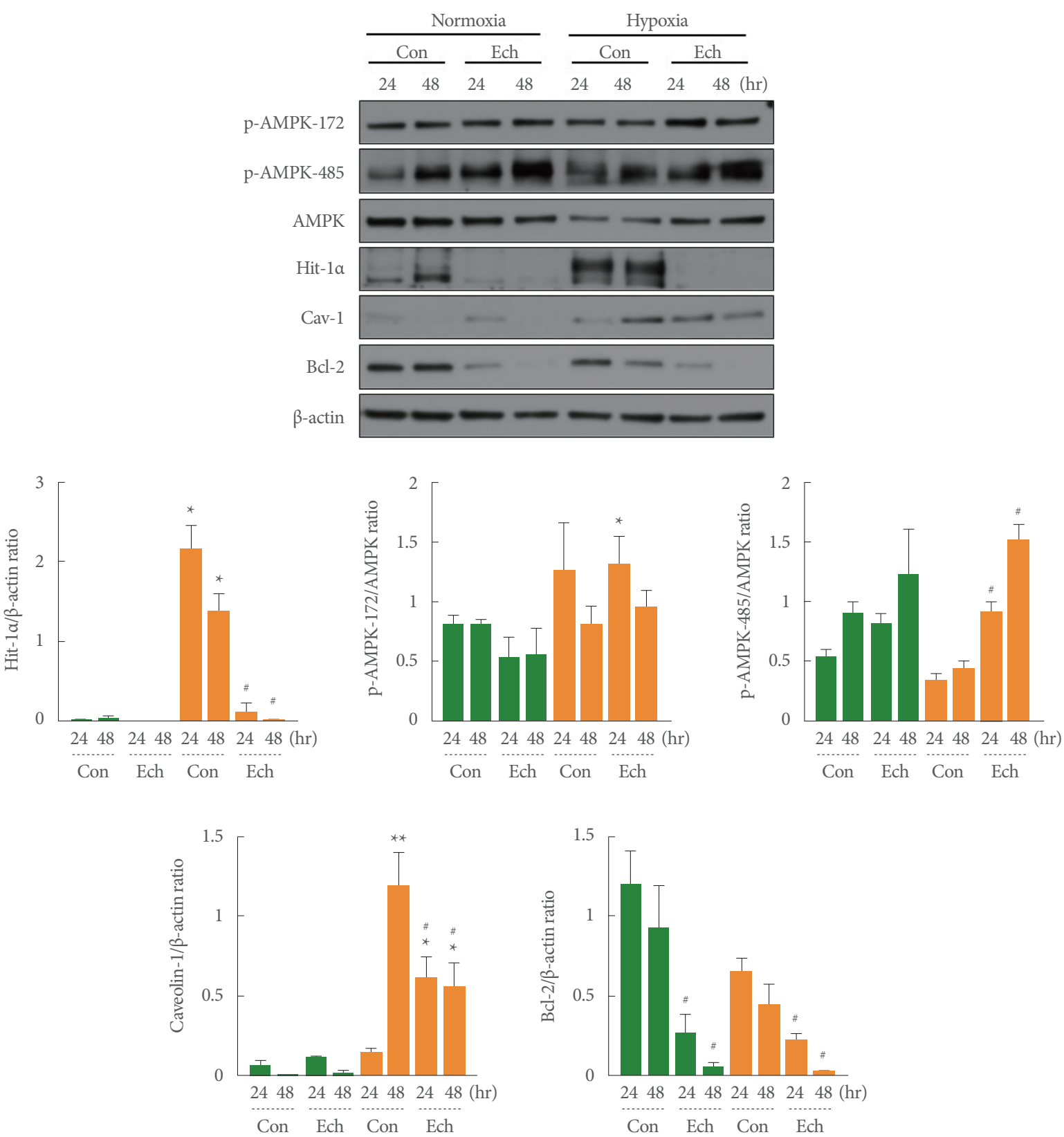

Fig. 6. Effects of the hypoxia-inducible factor 1 (HIF-1 1 ) selective inhibitor echinomycin (Ech, $2 \mathrm{nM}$ ) on the expressions and/or phospho-activities of adenosine monophosphate-activated protein kinase (AMPK), HIF-1 $\alpha$, caveolin-1 (Cav-1), and B-cell lymphoma 2 (Bcl-2) under normoxic and hypoxic conditions at 24 hours and 48 hours. The black and grey bars indicate the normoxia and hypoxia groups, respectively. The data presented are expressed as the mean \pm standard error of the mean $\left({ }^{*} \mathrm{P}<0.05,{ }^{* *} \mathrm{P}<0.01\right.$, vs. normoxia; ${ }^{*} \mathrm{P}<0.05$ vs. control group of normoxic or hypoxic condition).

tions, the triggering of Bcl-2 inhibition or the apoptosis pathway occurs only in the presence of AMPK activity and Cav-1 expression, and an excessive increase in AMPK activity suppresses hypoxia-induced Cav-1 expression (Fig. 3). Furthermore, the suppression of hypoxia-induced Bcl-2 expression was also reversed even in the cells treated with AMPK siRNA; this occurred due to the inhibition of AMPK activity, as shown in Fig. 7.

Noninvasive bladder urothelial cells, such as RT4 cells, are free of Cav-1 expression, even though this protein is highly ex- 

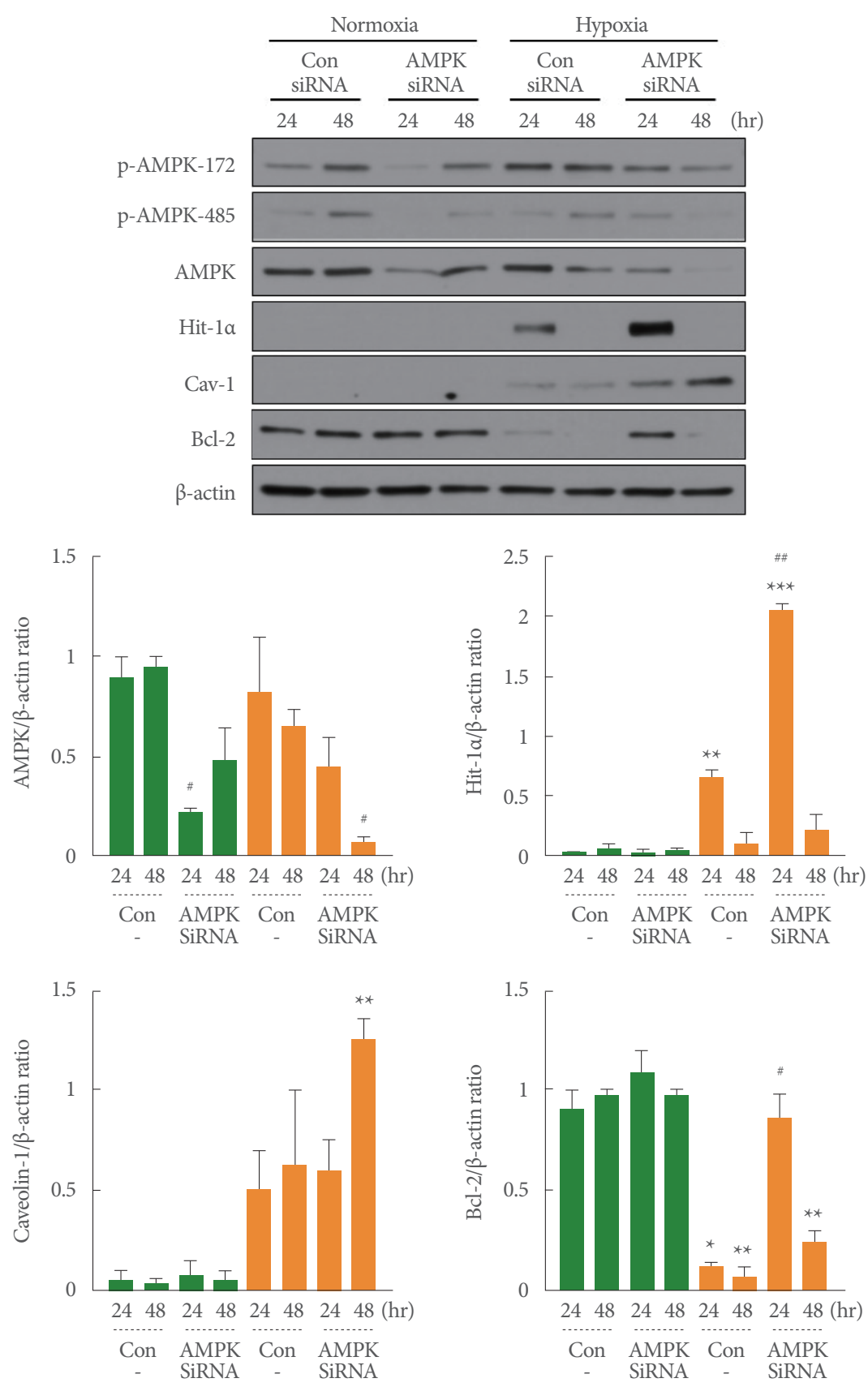

Fig. 7. Effects of transient silencing of adenosine monophosphate-activated protein kinase (AMPK) on hypoxia-inducible factor 1 (HIF-1a), caveolin-1 (Cav-1), and B-cell lymphoma 2 (Bcl-2) expression. Despite Cav-1 overexpression, hypoxia did not induce the suppression of Bcl-2 in cells pretreated with AMPK siRNA due to the loss of AMPK activity. The black and grey bars indicate the normoxia and hypoxia groups, respectively. The data presented are expressed as the mean \pm standard error of the mean $\left({ }^{\star} \mathrm{P}<0.05\right.$, ${ }^{* *} \mathrm{P}<0.01,{ }^{* \star} \mathrm{P}<0.001$ vs. normoxia; ${ }^{*} \mathrm{P}<0.05,{ }^{* *} \mathrm{P}<0.01$ vs. the control group of normoxic or hypoxic conditions).

pressed in smooth muscle cells [1,2,27-29]. Cav-1 expression has been shown to be upregulated under hypoxic conditions in both invasive and noninvasive tumor cells, such as clear-cell renal cell carcinoma [16], trophoblasts in preeclampsia [17], he- patocellular carcinoma [20], colon tissues in a mouse model of constitutively active HIF signals [30], and the noninvasive RT4 cells used in the present study. In these previous studies, HIFla was found to serve as a major transcription factor for Cav-1 
induction by acting on the conserved hypoxia response element under hypoxic conditions. In contrast to these results, the hypoxia-induced expression of HIF-1a resulted in a marked decrease of Cav-1 expression via the regulation of heat shock protein 90 [31]. Thus, the crosstalk between HIF-1 $\alpha$ and Cav-1 is still not fully understood.

The expression of Cav-1 in bladder urothelial cells is an important marker in distinguishing cancer grade, squamous differentiation, and cancer progression $[1,2,4]$. Cav-1 has been thought to mainly serve as a protein component of the caveolae structure in the cellular plasma membrane, as previously discussed [20]. Recently, however, the major Caveolin Research Group has reported various functional roles, including cell death and the growth of both normal and cancer cells [32-34]. In this regard, Liu et al. [21] reported the very interesting finding that Cav-1 can act as a coupling or sensitizing factor in the apoptotic signaling pathways of fibroblast (NIH/3T3) and invasive human bladder epithelial (T24) cells. This result is very similar to our finding that AMPK-induced cell apoptosis signaling activity can be induced in the presence of HIF-1a/Cav-1 expression. In particular, our results suggest that the expression of HIF-1 $\alpha$-mediated Cav-1 induced by hypoxia may play an important role in the pro-apoptotic pathway (by decreasing Bcl-2 expression) along with AMPK activity or activation.

Salani et al. [35] recently demonstrated that Cav-1 is essential to the antiproliferative activity of the AMPK activator metformin in non-small-cell lung cancer cells. As shown in Figs. 3 and 7, hypoxia-induced partial suppression of Bcl-2 was reversed not only by pretreatment with AMPK activators, but also by the blocking of AMPK with AMPK siRNA, suggesting that the hypoxia-induced suppression of anti-apoptotic Bcl-2 signaling is induced only when AMPK activity and Cav-1 expression are present concurrently. Therefore, Cav-1 may be a complementary factor for the apoptotic pathway induced by AMPK activation under hypoxic conditions. In other words, the inhibition of anti-apoptotic Bcl-2 signaling activity in RT4 cells exposed to hypoxic conditions suggests that HIF-1a-mediated Cav-1 induction and AMPK activity may play an interactive role in apoptotic cell death. In addition, the expression of Cav-1 was reduced in the presence of loss of HIF-1a expression due to treatment with echinomycin; thus, Bcl-2 did not differ from normoxia despite the presence of AMPK activity, probably due to the partial or lack of induction of Cav-1 and echinomycininduced apoptosis, as shown in Fig. 6.

In conclusion, we report a new finding that hypoxia-induced
AMPK activation can regulate HIF-1 $\alpha$-induced Cav-1 expression in Cav-1-free noninvasive RT4 bladder tumor cells. Additionally, we found that the substantial AMPK activity in hypoxia-induced suppression of the anti-apoptotic signaling pathway is exhibited only in cases of inducible Cav-1 expression. Thus, this AMPK activity is Cav-1-dependent.

\section{ORCID}

$\begin{array}{ll}\text { Tae Jin Cho } & 0000-0002-7865-6750 \\ \text { Da-Hyun Lee } & 0000-0001-7780-446 \mathrm{X} \\ \text { Bo-Hwa Choi } & 0000-0002-7907-8059 \\ \text { Helen K Shinn } & 0000-0003-1703-5501 \\ \text { Chang-Shin Park } & 0000-000106519-2857\end{array}$

\section{AUTHOR CONTRIBUTION STATEMENT}

- Conceptualization: CSP

- Data curation: CSP, TJC, BHC, HKS

- Formal analysis: TJC, DHL

- Funding acquisition: $C S P, B H C$

- Methodology: TJC, DHL, BHC

- Project administration: $C S P$

-Writing-original draft: TJC

-Writing-review \& editing: $C S P, H K S, B H C$

\section{REFERENCES}

1. Rajjayabun PH, Garg S, Durkan GC, Charlton R, Robinson MC, Mellon JK. Caveolin-1 expression is associated with high-grade bladder cancer. Urology 2001;58:811-14.

2. Fong A, Garcia E, Gwynn L, Lisanti MP, Fazzari MJ, Li M. Expression of caveolin-1 and caveolin- 2 in urothelial carcinoma of the urinary bladder correlates with tumor grade and squamous differentiation. Am J Clin Pathol 2003;120:93-100.

3. Thomas S, Overdevest JB, Nitz MD, Williams PD, Owens CR, Sanchez-Carbayo M, et al. Src and caveolin-1 reciprocally regulate metastasis via a common downstream signaling pathway in bladder cancer. Cancer Res 2011;71:832-41.

4. Liang W, Hao Z, Han JL, Zhu DJ, Jin ZF, Xie WL. CAV-1 contributes to bladder cancer progression by inducing epithelial-to-mesenchymal transition. Urol Oncol 2014;32:855-63.

5. Gleadle JM, Ratcliffe PJ. Hypoxia and the regulation of gene expression. Mol Med Today 1998;4:122-9.

6. De Marzo AM, Marchi VL, Epstein JI, Nelson WG. Proliferative 
inflammatory atrophy of the prostate: implications for prostatic carcinogenesis. Am J Pathol 1999;155:1985-92.

7. Vaupel P. The role of hypoxia-induced factors in tumor progression. Oncologist 2004;5:10-7.

8. Zhang H, Lu C, Fang M, Yan W, Chen M, Ji Y, et al. HIF-1a activates hypoxia-induced PFKFB4 expression in human bladder cancer cells. Biochem Biophys Res Commun 2016;476:146-52.

9. Towler MC, Hardie DG. AMP-activated protein kinase in metabolic control and insulin signaling. Circ Res 2007;100:328-41.

10. Wang W, Guan KL. AMP-activated protein kinase and cancer. Acta Physiol (Oxf) 2009;196:55-63.

11. Hallows KR, Mount PF, Pastor-Soler NM, Power DA. Role of the energy sensor AMP-activated protein kinase in renal physiology and disease. Am J Physiol Renal Physiol 2010;298: F1067-77.

12. Fukuyama Y, Ohta K, Okoshi R, Suehara M, Kizaki H, Nakagawa K. Hypoxia induces expression and activation of AMPK in rat dental pulp cells. J Dent Res 2007;86:903-7.

13. Emerling BM, Weinberg F, Snyder C, Burgess Z, Mutlu GM, Viollet $B$, et al. Hypoxic activation of AMPK is dependent on mitochondrial ROS but independent of an increase in AMP/ATP ratio. Free Radic Biol Med 2009;46:1386-91.

14. Jeong JH, Kang JH, Hwang SL, Cho HJ, Park KK, Park YY, et al. 4-O-methylascochlorin, methylated derivative of ascochlorin, stabilizes HIF-1a via AMPK activation. Biochem Biophys Res Commun 2011;406:353-8.

15. Hsu CCl, Wang CH, Wu LC, Hsia CY, Chi CW, Yin PH, et al. Mitochondrial dysfunction represses HIF-1a protein synthesis through AMPK activation in human hepatoma HepG2 cells. Biochim Biophys Acta 2013;1830:4743-51.

16. Wang Y, Roche O, Xu C, Moriyama EH, Heir P, Chung J, et al. Hypoxia promotes ligand-independent EGF receptor signaling via hypoxia-inducible factor-mediated upregulation of caveolin-1. Proc Natl Acad Sci U S A 2012;109:4892-97.

17. Jiang R, Cai J, Zhu Z, Chen D, Wang J, Wang Q, et al. Hypoxic trophoblast HMGB1 induces endothelial cell hyperpermeability via the TRL-4/caveolin-1 pathway. J Immunol 2014;193:5000-12.

18. Mao X, Wong SY, Tse EY, Ko FC, Tey SK, Yeung YS, et al. Mechanisms through which hypoxia-induced caveolin-1 drives tumorigenesis and metastasis in hepatocellular carcinoma. Cancer Res 2016;76:7242-53.

19. Cohen AW, Hnasko R, Schubert W, Lisanti MP. Role of caveolae and caveolins in health and disease. Physiol Rev 2004;84:1341-79.

20. Williams TM, Lisanti MP. Caveolin-1 in oncogenic transformation, cancer, and metastasis. Am J Physiol Cell Physiol 2005;288:C494506.
21. Liu J, Lee P, Galbiati F, Kitsis RN, Lisanti MP. Caveolin-1 expression sensitizes fibroblastic and epithelial cells to apoptotic stimulation. Am J Physiol Cell Physiol 2001;280:C823-35.

22. Sanchez-Carbayo M, Socci ND, Charytonowicz E, Lu M, Prystowsky M, Childs G, et al. Molecular profiling of bladder cancer using cDNA microarrays: defining histogenesis and biological phenotypes. Cancer Res 2002;62:6973-80.

23. Mungai PT, Waypa GB, Jairaman A, Prakriya M, Dokic D, Ball MK, et al. Hypoxia triggers AMPK activation through reactive oxygen species-mediated activation of calcium release-activated calcium channels. Mol Cell Biol 2011;31:3531-45.

24. Treins C, Murdaca J, Van Obberghen E, Giorgetti-Peraldi S. AMPK activation inhibits the expression of HIF-1alpha induced by insulin and IGF-1. Biochem Biophys Res Commun 2006;342:1197-202.

25. Park SJ, Lee KS, Kim SR, Chae HJ, Yoo WH, Kim DI, et al. AMPK activation reduces vascular permeability and airway inflammation by regulating HIF/VEGFA pathway in a murine model of toluene diisocyanate-induced asthma. Inflamm Res 2012;61:1069-83.

26. Zhou X, Chen J, Yi G, Deng M, Liu H, Liang M, et al. Metformin suppresses hypoxia-induced stabilization of HIF-1a through reprogramming of oxygen metabolism in hepatocellular carcinoma. Oncotarget 2016;7:873-84.

27. Polyák E, Boopathi E, Mohanan S, Deng M, Zderic SA, Wein AJ, et al. Alterations in caveolin expression and ultrastructure after bladder smooth muscle hypertrophy. J Urol 2009;182:2497-503.

28. Boopathi E, Gomes CM, Goldfarb R, John M, Srinivasan VG, Alanzi J, et al. Transcriptional repression of Caveolin-1 (CAV1) gene expression by GATA-6 in bladder smooth muscle hypertrophy in mice and human beings. Am J Pathol 2011;178:2236-51.

29. Sullivan MP, Cristofaro V, Radisavljevic ZM, Yalla SV. Regional distribution and molecular interaction of caveolins in bladder smooth muscle. BJU Int 2012;110:E1163-72.

30. Xie L, Xue X, Taylor M, Ramakrishnan SK, Nagaoka K, Hao C, et al. Hypoxia-inducible factor/MAZ-dependent induction of caveolin-1 regulates colon permeability through suppression of hosphor, leading to hypoxia-induced inflammation. Mol Cell Biol 2014;34: 3013-23.

31. Kannan A, Krishnan A, Ali M, Subramaniam S, Halagowder D, Sivasithamparam ND. Caveolin-1 promotes gastric cancer progression by up-regulating epithelial to mesenchymal transition by crosstalk of signalling mechanisms under hypoxic condition. Eur J Cancer 2014;50:204-15.

32. Martinez-Outschoorn UE, Trimmer C, Lin Z, Whitaker-Menezes D, Chiavarina B, Zhou J, et al. Autophagy in cancer associated fibroblasts promotes tumor cell survival: role of hypoxia, HIF1 in- 
duction and NFkB activation in the tumor stromal microenvironment. Cell Cycle 2010;9:3515-33.

33. Sotgia F, Martinez-Outschoorn UE, Howell A, Pestell RG, Pavlides S, Lisanti MP. Caveolin-1 and cancer metabolism in the tumor microenvironment: markers, models, and mechanisms. Annu Rev Pathol 2012;7:423-67.

34. Lisanti MP, Martinez-Outschoorn UE, Sotgia F. Oncogenes induce the cancer-associated fibroblast phenotype: metabolic symbiosis and "fibroblast addiction" are new therapeutic targets for drug discovery. Cell Cycle 2013;12:2723-32.

35. Salani B, Maffioli S, Hamoudane M, Parodi A, Ravera S, Passalacqua $\mathrm{M}$, et al. Caveolin-1 is essential for metformin inhibitory effect on IGF1 action in non-small-cell lung cancer cells. FASEB J 2012; 26:788-98. 